\title{
Cooperate to separate
}

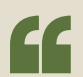

....interactions

between

different SMC

complexes

are being

shown to be

important for

the control of

chromosome

dynamics.
The complex mechanics of chromosome segregation during meiosis have recently been further clarified by Hong-Guo Yu and Douglas Koshland in Cell. They showed that the structural maintenance of chromosomes (SMC) complex condensin and the polo kinase $\mathrm{Cdc} 5$ are required for the removal of another SMC complex, cohesin, during the segregation of homologous chromosomes at anaphase I.

The successful segregation of homologous chromosomes during meiosis requires the coordination of sister-chromatid cohesion, condensation and recombination. The SMC complexes cohesin and condesin have crucial roles in these processes, so the authors studied their function and regulation using budding yeast.

Previous experiments had shown that yeast condensin mutants form chromosome bridges during meiosis because they are unable to properly segregate their homologous chromosomes during the first meiotic division (M1). The authors proposed that this is because condensin might be required to dissolve the recombinationdependent linkages that form between homologous chromosomes during meiosis efficiently. The molecular basis for homologue linkage and the potential role of condensin in this process were therefore examined.

Initially, the authors used green fluorescent protein (GFP)-tagged chromosomes to observe the effect on chromosome pairing of temperature-sensitive mutations in various condensin components. Their findings confirmed that a functional condensin complex is required to dissolve the homologue linkages on chromosome arms and telomeres.

In the same condensin-mutant strains, components of the cohesin complex were shown to remain localized at the bridged chromosomes long after anaphase I, when they would usually have been removed. Furthermore, introducing a mutation into a subunit of the cohesin complex or enhancing the ability of the cell to remove cohesin from chromosomes significantly reduced the amount of chromosome bridging in these cells. This indicates that it is the persistence of cohesin complexes on chromosomes that causes inappropriate homologue linkages, and that functional condensin is required for their removal.

The authors then tested the ability of two known cohesin regulators - the polo 


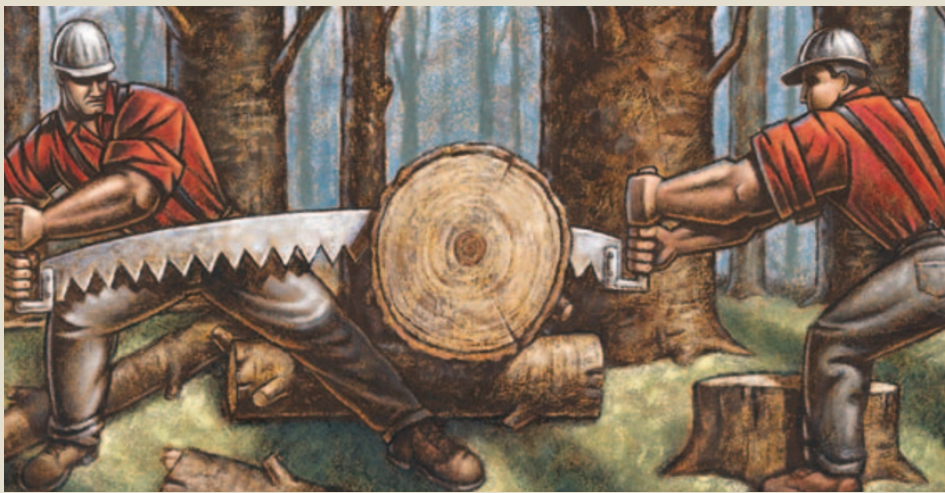

kinase $\mathrm{Cdc} 5$ and the Aurora-B homologue Ipl1 - to influence condensin-dependent cohesin removal. $\mathrm{Cdc} 5$ and, to a lesser extent, Ipl1 were both implicated in cohesin removal. Furthermore, condensin and $\mathrm{Cdc} 5$ were both required for hyperphosphorylation of the cohesin subunit $\operatorname{Rec} 8$, and the extent of $\operatorname{Rec} 8$ phosphorylation correlated with the extent of cohesin removal.

Cdc5 preferentially phosphorylates chromatin-bound cohesin during mitosis. So could condensin promote the Cdc5-mediated phosphorylation of cohesin by facilitating the chromosome binding of $\mathrm{Cdc} 5$ ? In keeping with this idea, the authors found that the association of $\mathrm{Cdc} 5$ with chromosomes is significantly perturbed during meiosis in condensin mutants. And increasing the level of $\mathrm{Cdc} 5$ during meiosis significantly ameliorated the effects of a condensin-subunit mutation.
So the condensin complex and the $\mathrm{Cdc} 5$ polo kinase are both required to facilitate the cohesin removal that allows the proper segregation of homologous chromosomes during meiosis. This reflects an emerging theme in chromosome biology, in which interactions between different SMC complexes are being shown to be important for the control of chromosome dynamics. Moreover, this mechanism also provides the opportunity for coupling chromosome dynamics to other meiotic events.

$$
\text { Lesley Cunliffe }
$$

\section{ORIGINAL RESEARCH PAPER}

Yu, H.-G. \& Koshland, D. Chromosome

morphogenesis: condensin-dependent cohesin removal during meiosis. Cell 123, 397-407

(2005)

WEB SITE

Douglas Koshland's laboratory:

http://www.ciwemb.edu/labs/koshland/

koshlandindex.html
Links:

\section{Cdc5}

http://db.yeastgenome.org/cgi-bin/locus.pl?locus=cdc5

Ipl1

http://db.yeastgenome.org/cgi-bin/locus.pl?locus=ipl1

Rec8

http://db.yeastgenome.org/cgi-bin/locus.pl?locus=rec8 